\title{
Tuberculosis treatment effect on T-cell interferon- $\gamma$ responses to Mycobacterium tuberculosis-specific antigens
}

\author{
C.B.E. Chee*, K.W. KhinMar*, S.H. Gan*, T.M. Barkham*, C.K. Koh*, \\ L. Shen and Y.T. Wang*
}

ABSTRACT: The hypothesis that T-cell interferon- $\gamma$ responses to Mycobacterium tuberculosisspecific antigens decline as disease activity diminishes with tuberculosis (TB) treatment has generated interest in the interferon- $\gamma$ release assays (IGRAs) as treatment-monitoring tools. We studied the effect of TB treatment on these responses as measured by the QuantiFERON-TB Gold In-tube (QFT-IT) and T-SPOT.TB ${ }_{\mathbb{R}}$ assays.

275 sputum culture-positive, HIV-uninfected pulmonary TB patients were tested with QFT-IT and T-SPOT.TB ${ }_{\circledR}$ at baseline, treatment completion and 6 months thereafter. The QFT-IT was also performed at the end of the intensive phase. The time-treatment effect on the qualitative and quantitative IGRA results was determined.

There were significant declines in the positivity rates and quantitative results of both IGRAs with treatment. The QFT-IT positivity rate was significantly lower than the T-SPOT.TB ${ }_{\mathbb{R}}$. The test reversion rate was significantly different for the two assays $\left(13.9 \%\right.$ for T-SPOT.TB ${ }_{\circledast}$ versus $39.2 \%$ for QFT-IT). $79 \%$ and $46 \%$ tested positive with T-SPOT.TB ${ }_{\mathbb{R}}$ and QFT-IT respectively at 6 months post-treatment completion. The kinetics of the quantitative responses was not significantly different between subjects with and without risk factors for disease relapse.

That a substantial proportion of patients remained test-positive after TB treatment would suggest a limited role of IGRAs as treatment monitoring tools.

KEYWORDS: Interferon- $\gamma$, T-cell, treatment, tuberculosis

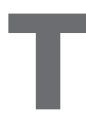

he interferon- $\gamma$ (IFN- $\gamma)$ release assays (IGRAs), the T-SPOT.TB® (Oxford Immunotec, Abingdon, UK), QuantiFERON$\mathrm{TB}_{\circledR}$ Gold (QFT-G) and the QuantiFERON-TB ${ }_{\circledast}$ Gold In-tube (QFT-IT) (both Cellestis, Carnegie, Victoria, Australia), have been granted regulatory approval in many countries to either replace [1] or supplement the tuberculin skin test as diagnostic tools in selected tuberculosis (TB) risk groups [2-4]. In addition to their increasingly established role in targeted latent TB infection (LTBI) testing [5-7], there has been interest in these assays as potential tools for monitoring disease activity. This is based on the hypothesis that the magnitude of the overnight T-cell responses to the Mycobacterium tuberculosis-specific early secreted antigen target (ESAT)-6, culture filtrate protein (CFP)-10 and Rv2654 (TB 7.7) antigens reflects mycobacterial antigen load, and hence disease activity [8]. Recent studies have shown significantly higher quantitative responses, as measured by the IGRAs in the active versus the latent TB state [9-11]. A few small, longitudinal studies utilising in-house or pre-commercial versions of the ELISpot-based assay have demonstrated declines in quantitative responses with active TB treatment [12-16]. A recent study in which the commercial T-SPOT.TB ${ }_{\mathbb{R}}$ assay was performed on frozen T-cells demonstrated a decline in the number of IFN- $\gamma$ producing RD-1specific T-cells with 24 weeks of treatment in 58 patients; there was however only a $10 \%$ reversion rate of the qualitative test result [17]. One study using the QFT-G in Japan, and one using the QFTIT in India showed weakening in these assay responses with treatment in active TB patients [18, 19]. In contrast, another study in India showed inconsistent changes in QFT-G IFN- $\gamma$ responses with treatment [20].

We conducted a prospective, head-to-head study to evaluate the effect of TB treatment on T-cell responses as measured by the T-SPOT.TB ${ }_{\circledR}$ and QFT-IT in pulmonary TB (pTB) patients treated under programme conditions at the Singapore TB Control Unit (TBCU). We further compared the
AFFILIATIONS

${ }^{*}$ Singapore Tuberculosis Control Unit,

\# Dept of Laboratory Medicine, Tan Tock Seng Hospital, and

'Statistics Unit, Dean's Office, Yong Loo Lin School of Medicine, National University of Singapore, Singapore.

CORRESPONDENCE

C.B.E. Chee

Tuberculosis Control Unit

144 Moulmein Road

308089

Singapore

E-mail: cynthia_chee@ttsh.com.sg

Received:

Sept 252009

Accepted after revision:

Nov 042009

First published online:

Nov 192009 
kinetics of these responses in subjects with and without risk factors predictive of disease relapse, namely, having a positive sputum culture at 2 months [21,22], lack of weight gain in the intensive phase in those who were underweight at baseline [23], and presence of cavity in the end-of-treatment chest radiograph [24].

\section{METHODS}

This study was approved by the Domain Specific Institutional Review Board of the National Healthcare Group, Singapore. The study population comprised patients of the TBCU, the national referral centre treating $\sim 60 \%$ of the country's TB cases. Subjects were prospectively recruited between April 2006 and May 2007. All participants gave informed consent.

Adult and adolescent patients who were sputum acid-fast bacilli (AFB) smear-positive, or who had clinical and chest radiographic findings consistent with bacteriologically positive pTB were considered for study enrolment. Patients with overall poor prognosis (e.g. the frail elderly, those with advanced malignancy), or who could not be followed-up for relapse (e.g. those not permanently residing in Singapore) were not recruited. Eligible patients were enrolled within 2 weeks of treatment commencement, at which time peripheral venous blood was drawn for both IGRAs (time A). At least two sputum specimens were obtained on separate days for AFB smears and TB culture and drug susceptibility testing (DST) prior to, or within 3 days of starting treatment. Human immunodeficiency virus (HIV) testing was routinely offered. Baseline random blood glucose and liver enzymes were routinely performed. Data on patient demographics, comorbidities, bacteriological status and radiological findings were captured. The treating physicians were blinded to the IGRA results. The patients were treated and monitored according to the centre protocol. At least one sputum specimen for TB culture and DST, and chest radiographs, were performed at the end of the intensive phase and at treatment completion. Baseline smear-positive patients underwent repeat sputum smear examination at completion of the intensive phase. 6-month short-course chemotherapy was the preferred treatment regimen, with the decision to use three or four drugs in the intensive phase individualised according to disease severity, the presence of risk factors for TB drug resistance and the patients' ability to tolerate the medications, as judged by the treating physician. Patients deemed at high risk for pyrazinamide hepatotoxicity were generally prescribed a 9-month regimen of rifampicin and isoniazid, with ethambutol (with or without streptomycin) in the first 2 months. Directly observed therapy (DOT) at the patient's nearest public health clinic is the standard treatment delivery mode under the national TB programme. DOT was administered daily during the intensive phase, and thrice weekly during the continuation phase. Blood was drawn for the QFT-IT at the end of the intensive phase (time B); and for both QFT-IT and T-SPOT.TB $B_{\mathbb{R}}$ at treatment completion (time C) and 6 months post-treatment completion (time D). Both assays were performed at the Tan Tock Seng Hospital Microbiology Laboratory, a 5-min walk from the TBCU.

\section{T-SPOT.TB ${ }_{\mathbb{R}}$ assay}

Blood was collected in dedicated cell preparation tube vacutainer tubes and sent to the laboratory at room temperature within $6 \mathrm{~h}$ of sampling. The assay was carried out according to the manufacturer's instructions. The test result was considered reactive if either or both of panel A (containing ESAT-6 antigen) or panel B (containing CFP-10 antigen) had six or more spots than the negative control and this number was at least twice the number of spots in the negative control.

\section{QFT-IT assay}

Blood was collected into three special heparinised $1 \mathrm{~mL}$ tubes; the "antigen" tube contained TB-specific stimulating antigens (ESAT-6, CFP-10 and TB7.7), the mitogen (positive control) tube contained phytohaemaglutinin, the third was a "nil" control tube. The assay was carried out according to the manufacturer's instructions. The test was considered positive if the IFN- $\gamma$ measured in the antigen tube minus the IFN- $\gamma$ measured in the nil tube was $\geqslant 0.35 \mathrm{IU} \cdot \mathrm{mL}^{-1}$ and was $\geqslant 25 \%$ of the value of the nil tube, and the nil value was $\leqslant 8 \mathrm{IU} \cdot \mathrm{mL}^{-1}$.

\section{Statistical analysis}

The analyses were performed using SAS 9.1 and SPSS 17. The patients' characteristics were summarised descriptively. The treatment effect was evaluated through the time effect from the start of treatment on the qualitative and quantitative IGRA results. A mixed model was utilised for analysing quantitative IGRA results, while a generalised estimation equation model for binary outcome using logic link was utilised for analysing qualitative IGRA results [25]. Bonferroni adjustment was applied when multiple comparisons were done, while the unadjusted p-value was reported. The Chi-squared test was used to compare the time A to time $C$ qualitative IGRA results between subjects with and without risk factors for relapse.

\section{RESULTS}

We recruited 350 subjects from April 2006 to May 2007. Of these, 286 were sputum culture-positive for $M$. tuberculosis complex (MTC). We have reported our comparison of the sensitivities of the baseline QFT-IT and T-SPOT.TB $B_{\mathbb{B}}$ results in 270 patients from this cohort [26]. For the present analysis, we excluded HIVpositive patients $(n=7)$, those with multidrug-resistant $T B(n=1)$ and those in whom baseline IGRA testing was performed $>14$ days after commencing treatment $(n=3)$. There were thus 275 subjects in this analysis. $263(95.6 \%)$ patients completed treatment; the vast majority $(95.4 \%)$ received DOT. $181(68.8 \%)$ subjects completed treatment in 6 months and $62(23.6 \%)$ in 9 months. The treatment duration for the remaining 20 patients ranged from 10 to 20 months. Four patients died (one in the intensive phase, three in the continuation phase), and eight permanently defaulted treatment. 32 (12.2\%) subjects did not return for evaluation at time D. A total of 231 subjects therefore completed the study protocol (fig. 1). The patient and disease characteristics at baseline are shown in table 1.

\section{Subjects with risk factors for disease relapse}

Figure 2 shows the distribution of the 58 patients according to the presence of risk factor(s) for relapse. 27 (10\%) of 272 subjects remained sputum culture-positive at the end of the intensive phase; all but one had pan-sensitive MTC grown. Of the 104 patients with body mass index (BMI) $<18.5 \mathrm{~kg} \cdot \mathrm{m}^{-2}$ at baseline, 26 failed to gain weight in the intensive phase. Twelve subjects had presence of cavity in their end-oftreatment chest radiograph. 


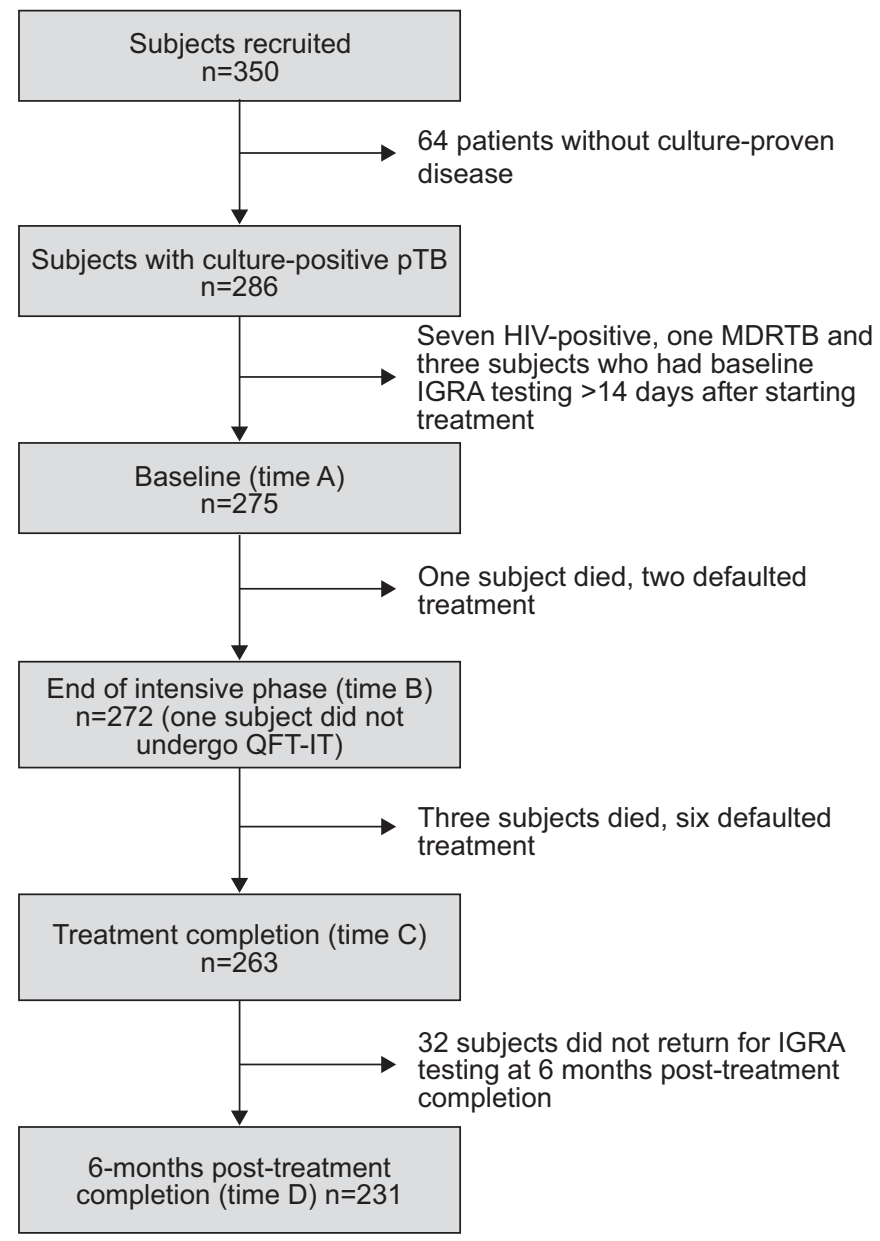

FIGURE 1. This chart outlines the flow of participants through the course of the study, and the number of subjects at each of the study time-points A to D. pTB: pulmonary tuberculosis; MDRTB: multidrug-resistant tuberculosis; IGRA: interferon- $\gamma$ release assay; QFT-IT: QuantiFERON-TBE Gold In-tube.

\section{IGRA results}

\section{Qualitative QFT-IT and T-SPOT.TB $₫$ results}

The indeterminate rate for QFT-IT was $2.2 \%$ (23 out of 1,040

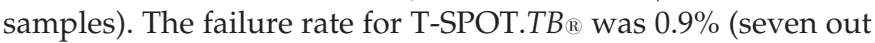
of 769 samples). These indeterminate and failed results were included in the analysis as "nonpositive" test results.

The QFT-IT was positive in $80.4 \%$ (221 out of 275$), 63.5 \%$ (172 out of 271$), 43.7 \%$ (115 out of 263 ) and $46.3 \%$ (107 out of 231 ) at times A, B, C and D respectively; the T-SPOT.TB $\mathbb{B}_{\text {was }}$ positive in $92.4 \%$ (254 out of 275 ), $82.5 \%$ (217 out of 263 ) and $79.2 \%$ (183 out of 231) at times A, C and D respectively (fig. 3). The positivity rates for both IGRAs changed significantly with time (and therefore treatment) from time A to $C$ ( $p<0.0001$ for both assays). This time/treatment effect was still significant after adjustment for age group, sex, race, BMI group, presence of cavity on chest radiograph, presence of diabetes, and sputum smear positivity ( $\mathrm{p}<0.0001$ for both assays). There was no significant change in the positivity rates for both assays from times $C$ to $D$ ( $p=0.66$ for QFT-IT, $p=0.186$ for T-SPOT.TB $\mathbb{R}$ ). The positivity rate of QFT-IT was significantly lower than that of the T-SPOT.TB $B_{\mathbb{B}}$ at all time-points $(\mathrm{p}<0.0001)$.

$\begin{array}{lc}\text { TABLE } 1 \text { Characteristics of subjects at baseline } \\ \begin{array}{l}\text { Age yrs } \\ \text { Male }\end{array} & 47.2(16.5-76.7) \\ \text { Ethnicity } & 203(73.8) \\ \text { Chinese } & \\ \text { Malay } & 186(67.6) \\ \text { Indian } & 65(23.6) \\ \text { Others } & 14(5.1) \\ \text { Presence of diabetes mellitus } & 10(3.6) \\ \text { Body mass index kg } \text { m }^{-2} & 99(36.0) \\ \text { Cigarette smoking } & 20.1(12.0-34.5) \\ \text { Current } & \\ \text { EX-smoker } & 115(41.8) \\ \text { Nonsmoker } & 38(13.8) \\ \text { Sputum smear } & 122(44.4) \\ \text { Positive } & \\ \text { Negative } & 210(76.4) \\ \text { Missing } & 63(22.9) \\ \text { Sputum MTC drug susceptibility } & 2(0.7) \\ \text { Pan-sensitive } & \\ \text { Mono-resistant to isoniazid } & 246(89.5) \\ \text { Mono-resistant to streptomycin } & 6(2.2) \\ \text { Mono-resistant to rifampicin } & 16(5.8) \\ \text { Poly-resistance (excluding MDRTB) } & 1(0.3) \\ \text { DST not done } & 5(1.8) \\ \text { Presence of cavity on initial chest } & 2(0.7) \\ \text { radiograph } & 140(50.9) \\ \end{array}$

Data are presented as mean (range) and $\mathrm{n}(\%)$, unless otherwise indicated MTC: Mycobacterium tuberculosis complex; MDRTB: multidrug-resistant tuberculosis; DST: drug susceptibility testing. $n=275$.

At treatment completion, $34(13.9 \%)$ of 244 baseline $\mathrm{T}$ SPOT.TB -positive subjects and $83(39.2 \%)$ of 212 baseline QFT-IT-positive subjects reverted their test results. The test reversion rate was significantly different for the two assays (McNemar test, $\mathrm{p}<0.0001$ ). The IGRA status for our subjects at baseline (time A) and treatment completion (time $\mathrm{C}$ ) according to the absence or presence of relapse risk factors is shown in table 2. The change of qualitative results of both assays between these two time-points was not significantly different between subjects with and without risk factors for relapse.

\section{Quantitative QFT-IT and T-SPOT.TB $\otimes$ results}

Pertaining to the QFT-IT assay, the mean IFN- $\gamma$ levels (antigen minus nil tube) were $4.87,2.75,1.95$, and $1.60 \mathrm{IU} \cdot \mathrm{mL}^{-1}$ at times A, B, C and D, respectively (fig. 4a). These levels changed significantly by time/treatment from time A to $C(p<0.0001)$, and the time/treatment effect was still significant after adjustment for age group, sex, race, BMI group, presence of cavity on chest radiograph, presence of diabetes, and sputum smear positivity $(p<0.0001)$. The mean IFN- $\gamma$ (antigen minus nil tube) levels did not change significantly from time $C$ to $D$ $(p=0.66)$. With respect to the T-SPOT.TB ${ }_{B}$ assay, the mean IFN- $\gamma$ producing spot-forming cells (SFCs) in response to ESAT-6 were 41,16 and $13 \mathrm{SFCs} / 2.5 \times 10^{5}$ peripheral blood mononuclear cells (PBMCs), while that to CFP-10 were 50, 38 and 27 SFCs $/ 2.5 \times 10^{5}$ PBMCs at times $A, C$ and $D$, respectively 


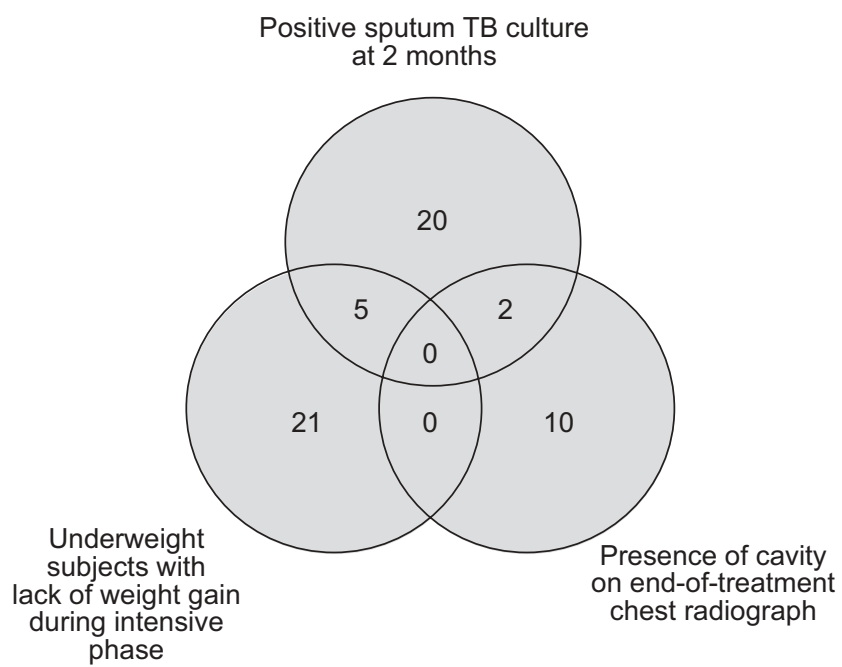

FIGURE 2. Venn diagram showing the distribution of the 58 subjects according to risk factor(s) associated with relapse of tubeculosis (TB) disease.

(fig. 4b). The mean responses to both antigens changed significantly by time $(\mathrm{p}<0.001)$, with the time/treatment effect still significant after adjustment for age group, sex, race, BMI group, presence of cavity in chest radiograph, presence of diabetes, and sputum smear results $(\mathrm{p}<0.0001)$. There was a significant overall difference between the mean quantitative response to ESAT-6 and CFP-10 $(\mathrm{p}<0.0001)$. The mean difference was 12.6 (95\% CI 8.38-16.86) SFCs $/ 2.5 \times 10^{5}$ PBMCs.

\section{Kinetics of the quantitative QFT-IT and T-SPOT.TB ${ }_{B}$ results of} subjects with and without relapse risk factors

The mean IFN- $\gamma$ levels (antigen minus nil tube) measured by the QFT-IT at times A, B, C and D were 6.11, 3.54, 1.80 and $1.99 \mathrm{IU} \cdot \mathrm{mL}^{-1}$ versus $4.68,2.57,1.98$, and $1.47 \mathrm{IU} \cdot \mathrm{mL}^{-1}$ in subjects with and without risk factors for relapse, respectively (fig. 5a). There was no significant difference in the kinetics of these responses between the two groups $(p=0.234)$. The mean responses to ESAT- 6 at times A, C and D were 45, 15 and 18

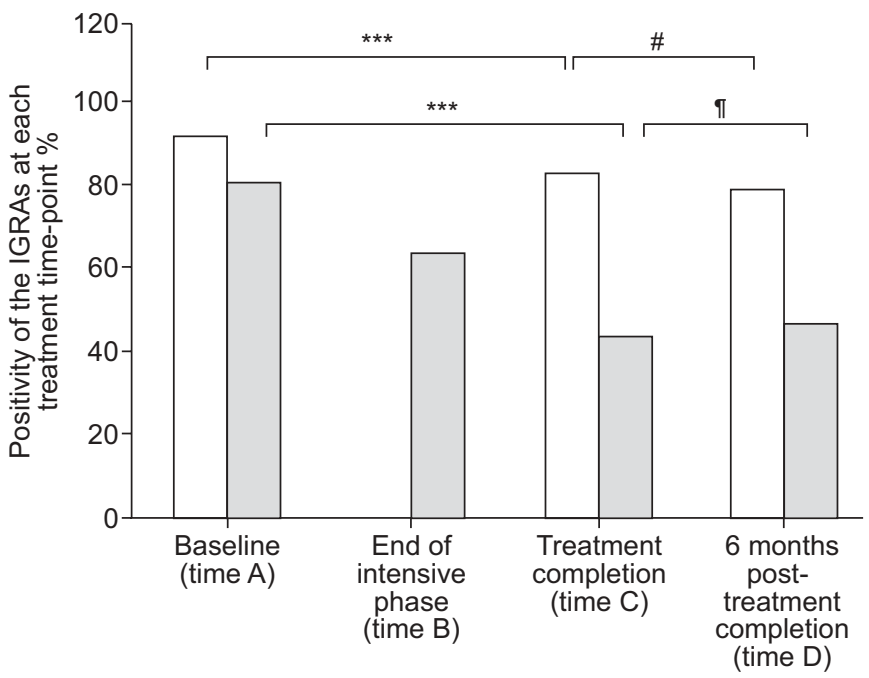

FIGURE 3. Percentage positivity of the interferon- $\gamma$ release assays (IGRAs), T-SPOT.TB $\mathbb{E}$ and QuantiFERON-TB $\mathbb{E}$ Gold In-tube (QFT-IT) assays at the study time-points. The QFT-IT and T-SPOT.TBв positivity rates declined significantly from baseline to treatment completion (time $A$ to $C)(p<0.0001)$, but not from time $C$ to $D$

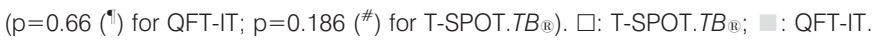

SFCs $/ 2.5 \times 10^{5}$ PBMCs versus 39,16 and 12 SFCs $/ 2.5 \times 10^{5}$ PBMCs in subjects with and without risk factors for relapse, respectively (fig. $5 b$ ). There was no significant difference in the kinetics of these responses between the two groups $(p=0.320)$. The mean responses to CFP-10 at times A, C and D were 47, 39 and 26 SFCs $/ 2.5 \times 10^{5}$ PBMCs versus 51,38 and 28 SFCs $/ 2.5 \times 10^{5}$ PBMCs in subjects with and without risk factors for relapse, respectively (fig. 5c). There was no significant difference in the kinetics of these responses between the two groups $(p=0.792)$.

\section{DISCUSSION}

We found significant declines in the qualitative and quantitative results of both IGRAs with TB treatment. There was a significant difference in the test reversion rate for the two assays $(13.9 \%$ for T-SPOT.TB ${ }_{\circledR}$ versus $39.2 \%$ for QFT-IT). There was no significant

TABLE 2 Time A to time C qualitative interferon- $\gamma$ release assay results in subjects and without risk factors for disease relapse

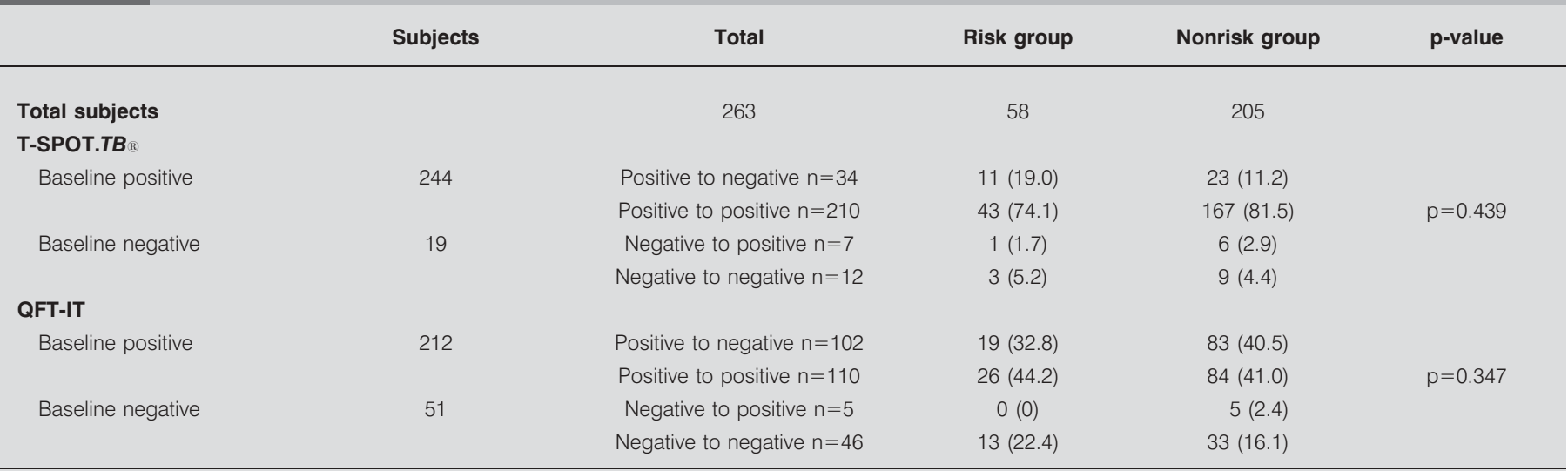

Data are presented as n or n (\%), unless otherwise indicated. QFT-IT: QuantiFERON-TB® Gold In-tube 

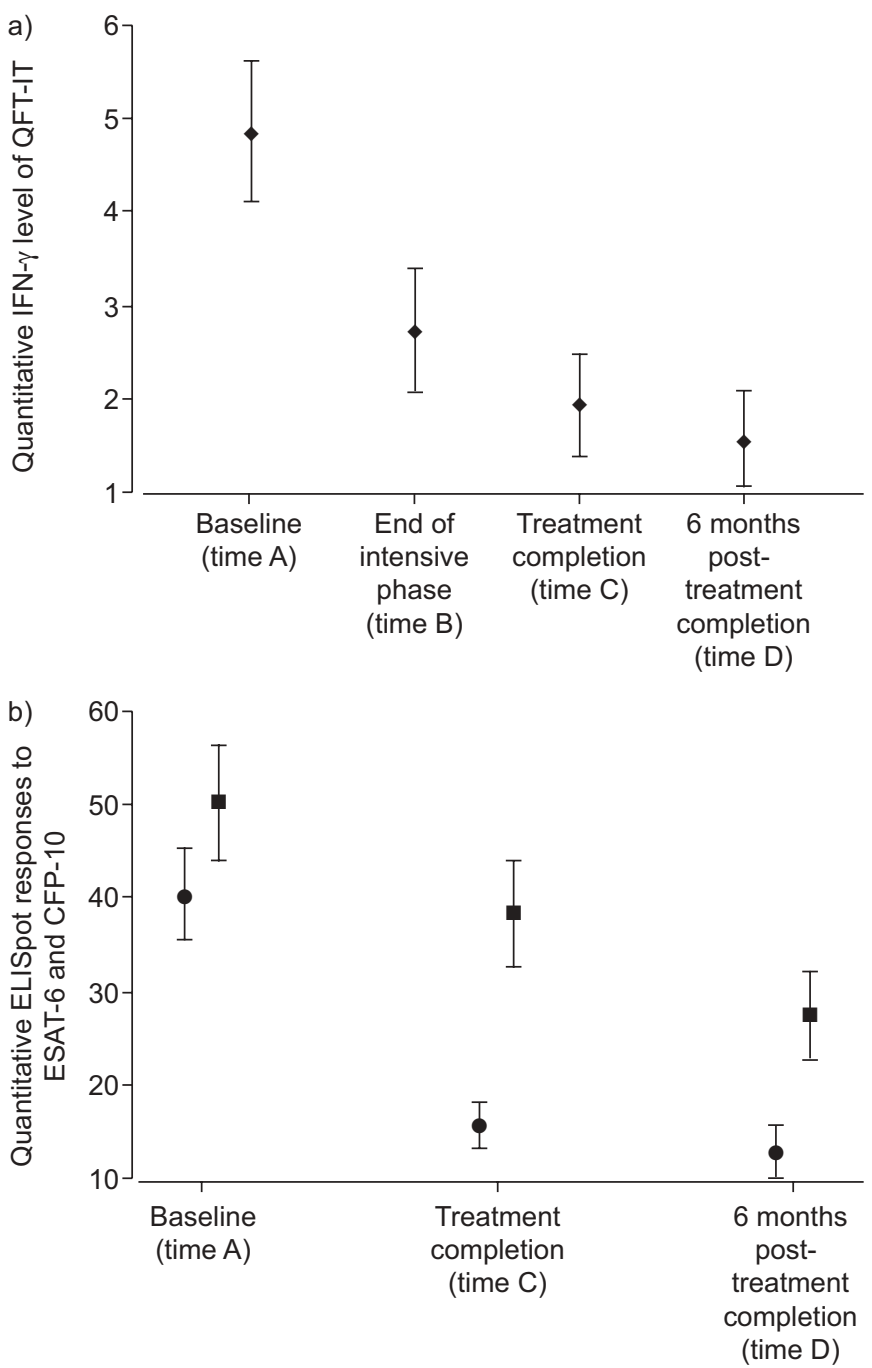

FIGURE 4. a) The mean interferon (IFN)- $\gamma$ levels (antigen minus nil tube) $\left(\mathrm{IU} \cdot \mathrm{mL}^{-1}\right.$ ) measured by the QuantiFERON-TB Gold In-tube (QFT-IT) assay were $4.87,2.75,1.95$, and $1.60 \mathrm{IU} \cdot \mathrm{mL}^{-1}$ at times A, B, C and D, respectively. These levels declined significantly from time $A$ to $C(p<0.0001)$, but not from time $C$ to $D$ $(p=0.66)$. b) The mean IFN- $\gamma$ producing spot-forming cells (SFCs) in response to early secreted antigen target (ESAT)-6 as measured by the T-SPOT.TB® assay were 41,16 and 13 SFCs $/ 2.5 \times 10^{5}$ peripheral blood mononuclear cells (PBMCs) at times $A, C$ and $D$, respectively. The mean responses to culture filtrate protein (CFP)-10 were 50, 38 and 27 SFCs $/ 2.5 \times 10^{5}$ PBMCs at times A, C and D, respectively. The responses to ESAT-6 and CFP-10 both declined significantly from time $A$ to $D$ $(p<0.001)$. There was a significant difference between the overall mean quantitative responses to ESAT-6 and CFP-10 ( $p<0.0001)$. - ESAT-6; $\mathbf{\square}$ : CFP-10.

change in the positivity rates of both assays from treatment completion to 6 months thereafter, with $79 \%$ and $46 \%$ subjects testing T-SPOT.TB $B_{\mathbb{R}}$-positive and QFT-IT-positive, respectively, at this time-point. The quantitative responses as measured by the T-SPOT.TB $B_{\circledR}$ (particularly that to CFP-10) declined further after treatment completion. The kinetics of these quantitative responses in subjects with and without risk factors for disease relapse was not significantly different.

Our findings are consistent with those of previous smaller studies on either IGRA. The present study adds to the literature as it has, to our knowledge, the largest treatment cohort of culture-proven TB patients in whom longitudinal testing with either of the commercial IGRAs has been reported, and is the first to compare the effect of active TB treatment on the TSPOT.TB $B_{\circledR}$ and QFT-IT in a head-to-head manner. The $44 \%$ QFTIT positivity rate of our subjects at treatment completion concurs with the $48 \%$ QFT positivity rates at 6 months of treatment reported by КоBASHI et al. [18] and KATIYAR et al. [19]. Our 13.9\% T-SPOT.TB $B$ reversion rate is marginally higher than the $10 \%$ reversion rate reported by RIBEIRO et al. [17]. The discrepancy in the test reversion rates and significantly lower positivity rate of the QFT-IT compared with the T-SPOT.TB $B_{\mathbb{B}}$ in our study is consistent with the lower sensitivity of the QFT-G/QFT-IT versus that of the T-SPOT.TB $B_{\circledR}$ reported in other head-to-head studies [27-29]. This may, in part, be attributed to the different manufacturers' choice of threshold values for positivity for the assays, which are primarily for LTBI detection. Using these cutoffs, a substantial proportion of our treated subjects remained IGRA-positive, especially with the T-SPOT.TB ${ }_{\mathbb{B}}$. Our study shows that qualitative IGRA results as interpreted according to the manufacturers' criteria would not be helpful for treatment monitoring, and that isolated positive test results cannot be interpreted meaningfully in previously treated TB patients. It has been suggested that persistently positive T-cell responses post-treatment may reflect on-going exposure to $M$. tuberculosis in the community [20]. While this may occur in high TB burden countries, Singapore's medium TB incidence rate of 35 out of 100,000 in 2006 and 2007 [30] would make this an unlikely explanation for our findings.

We previously showed, using the T-SPOT.TB $B_{\circledR}$ assay in TB contacts, that the number of IFN- $\gamma$ producing T-cells in response to CFP-10, but not that to ESAT-6 fell significantly with LTBI treatment [31]. This present study showed significant declines in T-cell responses to both ESAT- 6 and CFP-10 with active TB treatment. The overall quantitative responses to CFP-10 were significantly higher than those to ESAT-6, a similar finding to that in our TB contacts pre- and post-LTBI treatment. However, we observed a difference in the pattern of decline in the responses to these two antigens. The quantitative responses to CFP-10 demonstrated a steady decline from time A to time D, while that to ESAT-6 demonstrated a sharper decline from time A to time $C$, followed by a smaller degree of decline between time $\mathrm{C}$ and time $\mathrm{D}$. The pattern of decline seen with the response to ESAT-6 appeared to parallel that of the QFT-IT assay.

There is a pressing need for new tools to supplement sputum examination for the monitoring of $\mathrm{TB}$ treatment and the identification of patients at risk for poor treatment outcomes and relapse. Positive sputum cultures at 2 months (i.e. end of intensive phase) is a known risk factor for relapse or treatment failure [21, 22], and 2-month sputum bacteriological nonconversion is used as a surrogate marker for relapse and a standard endpoint in clinical trials evaluating new TB drug regimens. The utility of sputum TB cultures in the clinical and research arena is however limited by the time-lag of several weeks for result availability, and the difficulty in obtaining sputum specimens as patients' cough symptoms subside with TB treatment. More recently, it was shown that lack of weight gain of $>5 \%$ in the intensive phase in those who were underweight at baseline and presence of cavity in the end-of-treatment chest radiograph were independently associated with relapse risk [23, 24]. The notion that serial tracking of quantitative IGRA results could reflect 

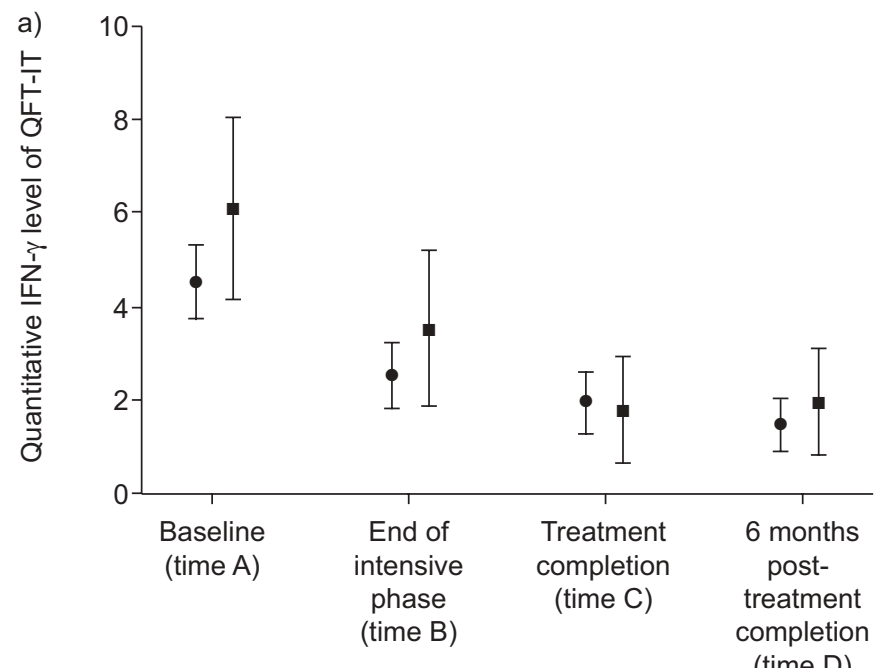

(time D)

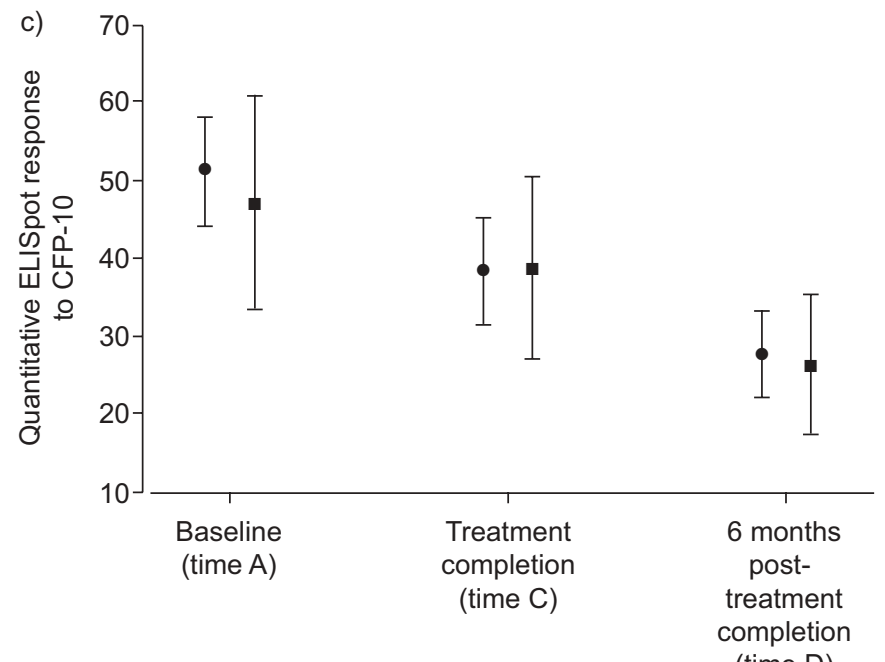

(time D)

treatment response or identify those at risk for poor treatment outcomes is attractive. This study showed significant declines in IFN- $\gamma$ levels and IFN- $\gamma$ producing RD-1-specific T-cells with treatment, supporting the hypothesis that these quantitative responses reflect disease activity. KATIYAR et al. [19] demonstrated that QFT-IT responses at 2 months of treatment significantly predicted the likelihood of remaining sputum culture-positive at the end of the intensive phase of treatment, suggesting the potential of these responses as treatmentmonitoring tools. Our study failed to show any significant difference between the kinetics of the quantitative T-cell responses as measured by either of the assays between subjects with and without independent risk factors predictive of disease relapse. Interestingly, however, we found small, nonsignificant increases in the IFN- $\gamma$ levels measured by the QFT-IT and the number of SFCs in response to ESAT-6 at 6 months posttreatment completion compared with end-of-treatment in subjects with risk factors for relapse. The importance, if any, of this finding remains to be further elucidated.

The strengths of this study are the relatively large cohort of culture-proven TB patients, the assurance of treatment adherence (the vast majority received DOT), the high $(95 \%)$ treatment

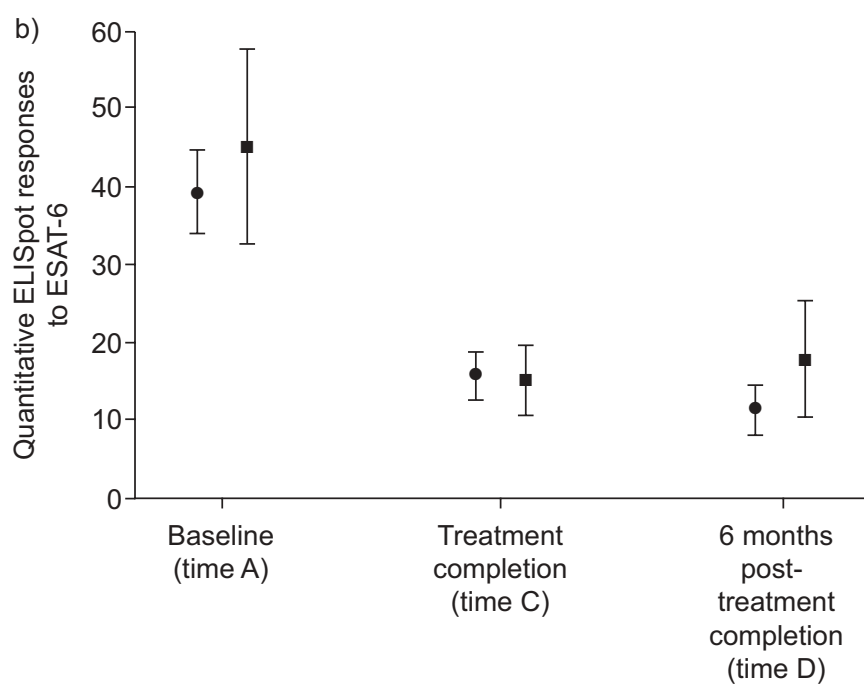

FIGURE 5. a) The mean interferon (IFN)- $\gamma$ levels (antigen minus nil tube) measured by the QuantiFERON-TB $B_{B}$ Gold In-tube (QFT-IT) assay at times A, B, C and $D$ were $6.11,3.54,1.80$ and $1.99 \mathrm{IU} \cdot \mathrm{mL}^{-1}$ versus $4.68,2.57,1.98$, and $1.47 \mathrm{IU} \cdot \mathrm{mL}^{-1}$ in subjects with and without risk factors for relapse, respectively. There was no significant difference in the kinetics of these responses between the two groups $(p=0.256)$. b) The mean responses to early secreted antigen target (ESAT)- 6 at times A, C and D were 45, 15 and 18 spot-forming cells (SFCs)/ $2.5 \times 10^{5}$ peripheral blood mononuclear cells (PBMCs) versus 39,16 and $12 \mathrm{SFCs}$ / $2.5 \times 10^{5}$ PBMCs in subjects with and without risk factors for relapse, respectively. There was no significant difference in the kinetics of these responses between the two groups $(p=0.320)$. c) The mean responses to culture filtrate protein (CFP)-10 at times A, C and D were 47,39 and 26 SFCs $/ 2.5 \times 10^{5}$ PBMCs versus 51,38 and 28 SFCs/2.5 $\times 10^{5}$ PBMCs in subjects with and without risk factors for relapse, respectively. There was no significant difference in the kinetics of these responses between the two groups $(p=0.792)$. Data are presented as mean \pm confidence interval. • : nonrisk; $\mathbf{\square}$ : risk.

completion rate, and low $(16 \%)$ drop-out rate of subjects over the study period. There were several study limitations. Funding constraints did not allow us to perform the T-SPOT.TB $\mathbb{\circledR}$ at the end of the intensive phase. However, as our T-SPOT.TB $\mathbb{B}$ positivity rate declined from $91 \%$ to $79 \%$ at treatment completion (versus $80 \%$ to $44 \%$ for the QFT-IT), it is unlikely that there would have been a significant difference in the T-SPOT.TB® positivity rate at the end of the intensive phase compared to baseline. Due to financial constraints, most patients had only one sputum TB culture performed at the end of the intensive phase. Our 10\% sputum nonconversion rate at this time-point is therefore likely to be an underestimation. Treatment regimens were also not strictly standardised, although standard short-course regimens were used in the majority, and $69 \%$ of our patients completed treatment in six months. Our study design was such that IGRA testing was performed at treatment completion, regardless of the duration of treatment, and not at pre-determined time-points (e.g. 6 months). We were thus unable to compare the IGRA results of those who responded well to treatment versus those who did not (and who consequently required treatment extension beyond the standard 6 months). Our cohort size was also insufficiently powered to study actual patient relapses, hence markers independently associated with relapse risk were used in our analysis. 
In conclusion, this study demonstrates the effect of TB treatment on T-cell IFN- $\gamma$ responses to M. tuberculosis-specific antigens as measured in a head-to-head manner with the QFT-IT and T-SPOT.TB ${ }_{\circledR}$ assays. We found significant declines in the qualitative and quantitative results of both IGRAs, with a significant difference in the positivity rates and test reversion rates between the assays. A substantial proportion of patients were still test-

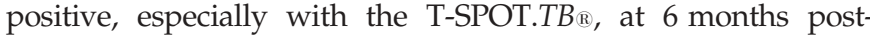
treatment completion. The kinetics of the quantitative T-cell responses was not significantly different between subjects with and without risk factors for disease relapse. Although our findings suggest that qualitative IGRA results as interpreted according to the manufacturers' criteria are not useful for treatment monitoring, the utility of serial quantitative T-cell responses as treatment monitoring tools may merit further evaluation.

\section{SUPPORT STATEMENT}

Funding source: Singapore Ministry of Health National Medical Research Council grant 1004/2005.

\section{STATEMENT OF INTEREST}

None declared.

\section{ACKNOWLEDGEMENTS}

We thank all the patients who participated in the study, Nursing Officer K-Y. Han, the medical and nursing staff of the TBCU, and Tan Tock Seng Hospital microbiology laboratory technicians A. Pereira and E.X. Yau. We thank S-C. Poh for critical comments.

\section{REFERENCES}

1 Centers for Disease Control and Prevention, Guidelines for using the QuantiFERON-TB Gold test for detecting Mycobacterium tuberculosis infection. United States. MMWR 2005; 54: 49-56.

2 National Institute for Health and Clinical Excellence 2006 Clinical Guidelines 33. Tuberculosis: clinical diagnosis and management of tuberculosis; and measures for its prevention and control. NICE, London, UK.

3 Canadian Tuberculosis Committee, Updated recommendations on interferon $\gamma$ release assays for latent tuberculosis infection. CCDR 2008; 34 (ACS-6):1-13.

4 Committee of Prevention, Japanese Society of Tuberculosis. [Guideline for the use of Quantiferon TB-2G.] Kekkaku 2006; 81: 393-397.

5 Pai M, Kalantri S, Dheda K. New tools and emerging technologies for the diagnosis of tuberculosis: Part I. Latent tuberculosis. Expert Rev Mol Diagn 2006; 6: 413-422.

6 Pai M, Zwerling A, Menzies D. Systematic review: T-cell-based assays for the diagnosis of latent tuberculosis infection: an update. Ann Intern Med 2008; 149: 177-184.

7 Richeldi L. Diagnosing latent tuberculosis infection: guess who's coming to dinner? Am J Respir Crit Care Med 2009; 180: 1-2.

8 Lalvani A. Counting antigen-specific T-cells: a new approach for monitoring response to tuberculosis treatment? Clin Infect Dis 2004; 38: 757-759.

9 Janssens J-P, Roux-Lombard P, Perneger T, et al. Quantitative scoring of an interferon- $\gamma$ assay for differentiating active from latent tuberculosis. Eur Respir J 2007; 30: 722-728.

10 Higuchi K, Harada N, Fukazawa K, et al. Relationship between whole-blood interferon-gamma responses and the risk of active tuberculosis. Tuberculosis 2008; 88: 244-248.

11 Chee CBE, Barkham TM, KhinMar KW, et al. Quantitative T-cell interferon- $\gamma$ responses to Mycobacterium tuberculosis-specific antigens in active and latent tuberculosis. Eur J Clin Microbiol Infect Dis 2009; 28: 667-670.
12 Pathan AA, Wilkinson KA, Klenerman P, et al. Direct ex-vivo analysis of antigen-specific IFN- $\gamma$-secreting CD4 T cells in Mycobacterium tuberculosis-infected individuals: associations with clinical disease state and effect of treatment. J Immunol 2001; 167: 5217-5225.

13 Lalvani A, Nagvenkar P, Udwadia Z, et al. Enumeration of T cells specific for RD 1-encoded antigens suggests a high prevalence of latent Mycobacterium tuberculosis infection in healthy urban Indians. J Infect Dis 2001; 183: 469-477.

14 Carrara S, Vincenti D, Petrosillo N, et al. Use of a T cell-based assay for monitoring efficacy of antituberculosis therapy. Clin Infect Dis 2004; 38: 754-756.

15 Nicol MP, Pienaar D, Wood K, et al. Enzyme-linked immunospot assay responses to early secretory antigenic target 6, culture filtrate protein 10, and purified protein derivative among children with tuberculosis: implications for diagnosis and monitoring of therapy. Clin Infect Dis 2005; 40: 1301-1308.

16 Aiken AM, Hill PC, Fox A, et al. Reversion of the ELISPOT test after treatment in Gambian tuberculosis cases. BMC Infect Dis 2006; 6: 66.

17 Ribeiro S, Dooley K, Hackman J, et al. T-SPOT.TB responses during treatment of pulmonary tuberculosis. BMC Infect Dis 2009; 9: 23.

18 Kobashi Y, Obase Y, Fukuda M, et al. Clinical reevaluation of the QuantiFERON TB-2G test as a diagnostic method for differentiating active tuberculosis from nontuberculous mycobacteriosis. Clin Infect Dis 2006; 43: 1540-1546.

19 Katiyar SK, Sampath A, Bihari S, et al. Use of the QuantiFERONTB Gold In-Tube test to monitor treatment efficacy in active pulmonary tuberculosis. Int J Tuberc Lung Dis 2008; 12: 1146-1152.

20 Pai M, Joshi R, Bandyopadhyay M, et al. Sensitivity of a wholeblood interferon-gamma assay among patients with pulmonary tuberculosis and variations in $\mathrm{T}$-cell responses during antituberculosis treatment. Infection 2007; 35: 98-103.

21 Aber VR, Nunn AJ. Factors affecting relapse following shortcourse chemotherapy. Bull Int Union Tuberc 1978; 53: 276-280.

22 The Tuberculosis Trials Consortium, Rifapentine and isoniazid once a week versus rifampicin and isoniazid twice a week for treatment of drug-susceptible pulmonary tuberculosis in HIV-negative patients: a randomised clinical trial. Lancet 2002; 360: 528-534.

23 Khan A, Sterling TR, Reeves R, et al. Lack of weight gain and relapse risk in a large tuberculosis treatment trial. Am J Respir Crit Care Med 2006; 174: 344-348.

24 Hamilton CD, Stout JE, Goodman PC, et al. The value of end-oftreatment chest radiograph in predicting pulmonary tuberculosis relapse. Int J Tuberc Lung Dis 2008; 12: 1059-1064.

25 Diggle PJ, Heagerty P, Liang K-Y, et al. Analysis of Longitudinal Data. Oxford, Oxford University Press, 2002.

26 Chee CBE, Gan SH, KhinMar KW, et al. Comparison of sensitivities of two commercial gamma interferon release assays for pulmonary tuberculosis. J Clin Microbiol 2008; 46: 1935-1940.

27 Ferrara G, Losi M, D'Amico R, et al. Use in routine clinical practice of two commercial blood tests for diagnosis of infection with Mycobacterium tuberculosis: a prospective study. Lancet 2006; 367: 1328-1334.

28 Lee JY, Choi HJ, Park I-N, et al. Comparison of two commercial interferon- $\gamma$ assays for diagnosing Mycobacterium tuberculosis infection. Eur Respir J 2006; 28: 24-30.

29 Dominquez J, Ruiz-Manzano J, De Souza-Galvao M, et al. Comparison of two commercially available $\gamma$ interferon blood tests for immunodiagnosis of tuberculosis. Clinical and Vaccine Immunology 2008; 15: 168-171.

30 Ministry of Health, Singapore. Communicable disease surveillance report in Singapore 2007, Singapore 2008. Singapore, Ministry of Health, 2008.

31 Chee CBE, KhinMar KW, Gan SH, et al. Latent tuberculosis infection treatment and T-cell responses to Mycobacterium tuberculosis-specific antigens. Am J Respir Crit Care Med 2007; 175: 282-287. 